\title{
Oral Vancomycin Prophylaxis Is Highly Effective in Preventing Clostridium difficile Infection in Allogeneic Hematopoietic Cell Transplant Recipients
}

\author{
Alex Ganetsky, ${ }^{1}$ Jennifer H. Han, ${ }^{2,3}$ Mitchell E. Hughes, ${ }^{1}$ Daria V. Babushok, ${ }^{4}$ Noelle V. Frey, ${ }^{4}$ Saar I. Gill, ${ }^{4}$ Elizabeth 0. Hexner, ${ }^{4}$ Alison W. Loren, ${ }^{4}$ \\ Selina M. Luger, ${ }^{4}$ James K. Mangan, ${ }^{4}$ Mary Ellen Martin, ${ }^{4}$ Jacqueline Smith, ${ }^{4}$ Craig W. Freyer, ${ }^{1}$ Cheryl Gilmar, ${ }^{3}$ Mindy Schuster, ${ }^{2}$ \\ Edward A. Stadtmauer, ${ }^{4}$ and David L. Porter ${ }^{4}$ \\ ${ }^{1}$ Department of Pharmacy, Hospital of the University of Pennsylvania, ${ }^{2}$ Division of Infectious Diseases, Department of Medicine, Perelman School of Medicine at the University of Pennsylvania, \\ ${ }^{3}$ Department of Healthcare Epidemiology, Infection Prevention and Control, Hospital of the University of Pennsylvania, and ${ }^{4}$ Blood and Marrow Transplantation Program, Abramson Cancer Center \\ and the Division of Hematology and Oncology, Perelman School of Medicine at the University of Pennsylvania, Philadelphia
}

Background. Clostridium difficile infection (CDI) is a leading cause of infectious complications in allogeneic hematopoietic cell transplant recipients (alloHCT). We sought to evaluate whether prophylactic oral vancomycin reduces the incidence of CDI in alloHCT recipients.

Methods. We conducted a retrospective cohort study to examine the effectiveness of CDI prophylaxis with oral vancomycin, as compared to no prophylaxis, in 145 consecutive adult alloHCT recipients at the University of Pennsylvania between April 2015 and November 2016. Patients received oral vancomycin $125 \mathrm{mg}$ twice daily, starting on admission and continuing until discharge. The primary outcome of interest was the association between oral vancomycin prophylaxis and CDI diagnosis. Secondary outcomes included graft-versus-host disease (GVHD) and relapse.

Results. There were no cases of CDI in patients that received prophylaxis (0/90, 0\%), whereas 11/55 (20\%) patients who did not receive prophylaxis developed CDI $(P<.001)$. Oral vancomycin prophylaxis was not associated with a higher risk of acute, grades 2-4 GVHD (subhazard ratio [sHR] 1.59; 95\% confidence interval [CI] 0.88-2.89; $P=.12$ ), acute, grades 3-4 GVHD (sHR 0.65; 95\% CI $0.25-1.66 ; P=.36$ ), or acute, grades $2-4$ gastrointestinal GVHD (sHR $1.95 ; 95 \%$ CI $0.93-4.07 ; P=.08$ ) at day 180 post-transplant. No associations between oral vancomycin and relapse or survival were observed.

Conclusions. Prophylaxis with oral vancomycin is highly effective in preventing CDI in alloHCT recipients without increasing the risk of graft-versus-host disease or disease relapse. Further evaluation via a prospective study is warranted.

Keywords. vancomycin; Clostridium difficile; stem cell transplant.

Infection remains a major cause of morbidity and mortality following alloHCT [1]. Among the most common infectious complications is Clostridium difficile infection (CDI), which occurs in up to $33 \%$ of alloHCT recipients and has been identified as an independent risk factor for increased mortality [2, 3]. AlloHCT recipients are particularly susceptible to $\mathrm{CDI}$, owing to a multitude of factors, including extensive exposure to broad-spectrum antibiotics, a prolonged period of neutropenia, lengthy hospitalizations, and gastrointestinal mucosal damage from chemotherapy and/or irradiation $[4,5]$.

Received 8 June 2018; editorial decision 10 September 2018; accepted 20 September 2018; published online September 26, 2018.

Correspondence: J. H. Han, Division of Infectious Diseases, Department of Medicine, Perelman School of Medicine at the University of Pennsylvania, 734 Blockley Hall, 423 Guardian Drive, Philadelphia, PA 19104 (jennifer.han@uphs.upenn.edu).

Clinical Infectious Diseases ${ }^{\circledR} \quad$ 2019;68(12):2003-9

(C) The Author(s) 2018. Published by Oxford University Press for the Infectious Diseases Society of America. All rights reserved. For permissions, e-mail: journals.permissions@oup.com. DOI: 10.1093/cid/ciy822
Previous studies have also shown that CDI diagnosis early in the alloHCT course increases the risk of graft-versus-host disease (GVHD), including, specifically, GVHD of the gastrointestinal (GI) tract, an immunologic complication mediated predominantly by donor-derived $\mathrm{T}$ cells that react against the recipient's host tissues $[6,7]$. The association between CDI and GVHD is alarming, as GVHD is the leading cause of non-relapse mortality in alloHCT recipients [8]. Early CDI during alloHCT has been found to increase the risk of GI GVHD by more than 3-fold $[6,9]$. The mechanistic interplay between CDI and GVHD is hypothesized to result from CDI-induced, localized intestinal inflammation, along with a reduction in intestinal microbial diversity. Changes in the intestinal microbiota appear to have significant consequences in the context of alloHCT outcomes, such as GVHD and disease relapse [10].

The high incidence and the potentially severe consequences of CDI in alloHCT recipients highlight the urgently-needed but difficult-to-achieve goal of mitigating the risk of CDI in this patient population. As such, we aimed to evaluate the effectiveness of oral vancomycin prophylaxis in alloHCT recipients, as well 
as to examine the associations between oral vancomycin prophylaxis and other important clinical outcomes, such as GVHD, disease relapse, and survival.

\section{METHODS}

We conducted a retrospective cohort study to examine the effectiveness of CDI prophylaxis with oral vancomycin, compared to no prophylaxis, in 145 consecutive adults undergoing alloHCT at the Hospital of the University of Pennsylvania between April 2015 and November 2016. We initiated a pilot program of CDI prophylaxis with oral vancomycin for all alloHCT recipients, starting in December 2015, as a quality-improvement initiative. Patients received oral vancomycin at $125 \mathrm{mg}$ twice daily, starting on the day of inpatient admission for alloHCT and continuing until the day of discharge. Prior to the initiation of this pilot, pharmacologic prophylaxis for CDI was not administered. The primary exposure was receipt of oral vancomycin prophylaxis, which occurred during the time period between December 2015 and November 2016. These exposed patients were compared to unexposed patients between April 2015 and December 2015.

The primary outcome of interest was the association between CDI prophylaxis with oral vancomycin and a diagnosis of CDI during the time from inpatient admission for alloHCT to hospital discharge. C. difficile testing on stool samples during the entire study period was performed by the Hospital of the University of Pennsylvania's Clinical Microbiology Laboratory using a commercial enzyme immunoassay for the detection of toxins A and B and glutamate dehydrogenase (C Diff Quik Check Complete, Alere). Samples which were negative for toxins $\mathrm{A}$ and $\mathrm{B}$ but positive for glutamate dehydrogenase were subsequently tested using polymerase chain reaction (PCR) for toxin genes (BD MAX Cdiff Assay, Becton Dickinson). All alloHCT recipients were admitted to the same inpatient unit, dedicated to the care of HCT patients. All rooms have high-energy particulate air filtration and positive pressure, We are not aware of any practice patterns or changes in cleaning procedures that were made during the study period.

Secondary endpoints included acute, grades 2-4 GVHD; acute, grades 3-4 GVHD; chronic GVHD; relapse; non-relapse mortality (NRM); GVHD-free, relapse-free survival; and overall survival (OS). In addition, the incidence of vancomycin-resistant enterococcus (VRE) bloodstream infections was evaluated, given the previously described association between oral vancomycin exposure and VRE [11]. No routine screening for VRE colonization was performed during the study period. Acute GVHD was graded according to the modified Glucksberg criteria [12]. Chronic GVHD was graded according to the National Institutes of Health Consensus Criteria [13]. Disease relapse was defined as morphological, cytogenetic, or radiological evidence of a disease demonstrating pre-transplant characteristics.
We used the Disease Risk Index stratification system to classify patients according to disease type and status [14].

The following variables were collected using a medical records review: age, sex, disease type, donor source, degree of HLA match, conditioning intensity, disease risk index, CDI history during the prior year, GVHD prophylaxis regimen, and broad-spectrum antibiotic exposure both within 30 days prior to alloHCT and post-transplant until either development of CDI or 30 days after alloHCT, whichever occurred first.

Characteristics were compared between the 2 groups, with categorical variables compared using the $\mathrm{X}^{2}$ or Fisher exact test and continuous variables using the Wilcoxon rank-sum test. Primary and secondary outcome analyses were conducted using survival analyses to determine the association between oral vancomycin prophylaxis and the time to development of the event of interest. Time 0 for all outcomes was defined as the date of alloHCT. Acute GVHD outcomes were censored at 6 months for patients who did not reach the event of interest. All other secondary endpoints were censored at 12 months for patients who did not reach the event of interest.

Competing risks regression was used for the GVHD outcomes, relapse, and NRM. For the GVHD and relapse outcomes, death was modeled as a competing risk. For the NRM outcome, relapse was modeled as a competing risk. Subhazard ratios (sHR) and 95\% confidence intervals (CIs) were calculated to evaluate the strength of any association. For the outcome of OS, a Cox proportional hazards regression analysis was performed, with the calculation of hazard ratios (HR) and 95\% CIs. Potential multivariable models were developed, beginning with the primary exposure of interest: receipt of oral vancomycin prophylaxis. A manual stepwise selection procedure was used, with variables with a $P$ value of $<.20$ on bivariable analysis considered as candidate variables and maintained in the final model if their inclusion was statistically significant on likelihood ratio testing. All calculations were performed using Stata v15.1 (StataCorp LC, College Station, TX). For all calculations, a 2 -tailed $P$ value <.05 was considered to be significant. This study was approved by the Institutional Review Board of the University of Pennsylvania.

\section{RESULTS}

A total of 145 patients were included during the study period, of which 90 (62\%) were in the exposed group (ie, received oral vancomycin prophylaxis). An additional 3 patients were unable to receive vancomycin and were excluded from the analysis (2 patients with drug intolerance and 1 patient with a history of vancomycin allergy). Patient and disease-related characteristics are reported in Table 1 . Patients were generally high risk by virtue of older age (median 55 years), a high disease risk index score (high/very high: $45.5 \%$ ), and a hematopoietic cell

2004 • CID 2019:68 (15 June) • Ganetsky et al 


\begin{tabular}{|c|c|c|c|}
\hline Characteristic & $\begin{array}{l}\text { Oral Vancomycin Prophylaxis } \\
\qquad(n=90)\end{array}$ & $\begin{array}{l}\text { No Prophylaxis } \\
\quad(n=55)\end{array}$ & $P$ Value \\
\hline Recipient age, in years, median (range) & $59(20-73)$ & $57(19-72)$ & .30 \\
\hline Diagnosis, $\mathrm{n}(\%)$ & & & .42 \\
\hline Acute myeloid leukemia & $47(52.2)$ & 35 (63.6) & \\
\hline Acute lymphoblastic leukemia & $15(16.7)$ & $6(10.9)$ & \\
\hline Myelodysplastic syndromes & $13(14.4)$ & $7(12.7)$ & \\
\hline Chronic myeloid leukemia & $4(4.4)$ & $1(1.8)$ & \\
\hline Myelofibrosis & $2(2.2)$ & $2(3.6)$ & \\
\hline Other $^{\mathrm{a}}$ & $9(10)$ & $4(7.3)$ & \\
\hline Donor source, $\mathrm{n}(\%)$ & & & .54 \\
\hline Sibling & $27(30.0)$ & $14(25.4)$ & \\
\hline Unrelated & $49(54.4)$ & $35(63.6)$ & \\
\hline Double umbilical cord blood & $8(8.9)$ & $4(7.3)$ & \\
\hline Haploidentical & $6(6.7)$ & 2 (3.6) & \\
\hline Conditioning intensity, $\mathrm{n}(\%)$ & & & .36 \\
\hline Myeloablative & $48(53.3)$ & 25 (45.5) & \\
\hline Reduced-intensity & $42(46.7)$ & $30(54.5)$ & \\
\hline Graft source, n (\%) & & & .86 \\
\hline Peripheral blood & $58(64.4)$ & $38(69.1)$ & \\
\hline Bone marrow & $24(26.7)$ & $13(23.6)$ & \\
\hline Double umbilical cord blood & $8(8.9)$ & $4(7.3)$ & \\
\hline GVHD prophylaxis, n (\%) & & & .09 \\
\hline TAC/MTX & $59(65.6)$ & $32(58.2)$ & \\
\hline TAC/MMF & $18(20)$ & $7(12.7)$ & \\
\hline Other $^{\mathrm{b}}$ & $13(14.4)$ & 16 (29) & \\
\hline History of Clostridium difficile infection, $n(\%)$ & $14(15.6)$ & $5(9.1)$ & .32 \\
\hline
\end{tabular}

$\mathrm{N}=145$.

Abbreviations: GVHD, graft-versus-host disease; MMF, mycophenolate mofetil; MTX, methotrexate; TAC, tacrolimus.

${ }^{a}$ Other includes aplastic anemia $(n=1)$, chronic lymphocytic leukemia $(n=3)$, Hodgkin's lymphoma CDI $(n=1)$, multiple myeloma ( $\left.n=2\right)$, and non-Hodgkin's lymphoma ( $\left.=6\right)$.

${ }^{b}$ Other includes cyclophosphamide $(n=1)$, cyclosporine/mycophenolate mofetil $(n=5)$, tacrolimus/methotrexate/bortezomib $(n=5)$, tacrolimus/mycophenolate mofetil/cyclophosphamide

$(n=10)$, and tacrolimus/methotrexate/maraviroc $(n=8)$.

transplantation-specific comorbidity index score (score $\geq 3$ : $56.3 \%)$.

There were no significant differences in patient, disease, or transplant characteristics between the intervention and control groups. Gram-negative and gram-positive antibiotic exposure within 30 days prior to alloHCT was similar between the groups (Table 2). However, metronidazole use was greater in the control group compared with the intervention group (7\% vs $0 \%$;
$P=.02)$. There were no differences in the use of gram-negative antibiotics, gram-positive antibiotics, or metronidazole between the intervention and control groups during the time at risk (Table 3).

\section{Clostridium difficile Infection}

There were no cases of CDI in patients that received oral vancomycin prophylaxis $(0 / 90 ; 0 \%)$ whereas $11 / 55(20 \%)$ patients

Table 2. Type of Antibiotic Exposure 30 Days Prior to Allogeneic Hematopoietic Cell Transplantation

\begin{tabular}{|c|c|c|c|}
\hline Antibiotic & $\begin{array}{l}\text { Oral Vancomycin Prophylaxis } \\
\qquad(n=90)\end{array}$ & $\begin{array}{l}\text { No Prophylaxis } \\
\quad(n=55)\end{array}$ & $P$ Value \\
\hline Gram-negative antibiotics, ${ }^{a} \mathrm{n}(\%)$ & $17(19)$ & $9(16)$ & .82 \\
\hline Gram-negative antibiotic days, median (IQR) & $4(2,6)^{b}$ & $2(2,4)^{b}$ & .57 \\
\hline Intravenous vancomycin, $\mathrm{n}(\%)$ & $7(8)$ & $6(11)$ & .56 \\
\hline Intravenous vancomycin days, median (IQR) & $0(0,1)^{b}$ & $0(0,1)^{b}$ & .54 \\
\hline Metronidazole, n (\%) & $0(0)$ & $4(7)$ & .02 \\
\hline Metronidazole days, median (IOR) & - & $1.5(1,2)$ & \\
\hline
\end{tabular}

Abbreviations: IQR, interquartile range.

${ }^{a}$ Gram-negative antibiotics included cefepime, piperacillin/tazobactam, meropenem, ceftazidime, aztreonam, and levofloxacin .

${ }^{b}$ Reported as only including patients who received the antibiotic, given overall low numbers. 


\begin{tabular}{|c|c|c|c|}
\hline Antibiotic & $\begin{array}{l}\text { Oral Vancomycin Prophylaxis } \\
\qquad(N=90)\end{array}$ & $\begin{array}{c}\text { No Prophylaxis } \\
(\mathrm{N}=55)\end{array}$ & $P$ Value \\
\hline Gram negative antibiotics, ${ }^{a} \mathrm{n}(\%)$ & $68(76)$ & $39(71)$ & .54 \\
\hline Gram-negative antibiotic days, median (IQR) & $12(2,16)$ & $9(0,15)$ & .23 \\
\hline Intravenous vancomycin, n (\%) & $42(47)$ & $21(38)$ & .32 \\
\hline Intravenous vancomycin days, median (IQR) & $0(0,4)$ & $0(0,6)$ & .72 \\
\hline Metronidazole, n (\%) & $6(7)$ & $9(16)$ & .09 \\
\hline Metronidazole days, median (IOR) & $3.5(2,4)$ & $6(3,12)$ & .053 \\
\hline
\end{tabular}

Time of antibiotic exposure after alloHCT was assessed until either development of CDI or 30 days after transplant, whichever occurred first. Abbreviations: alloHCT, allogeneic hematopoietic cell transplantation; CDI, Clostridium difficile infection; IQR, interquartile range

${ }^{a}$ Gram-negative antibiotics included cefepime, piperacillin/tazobactam, meropenem, ceftazidime, aztreonam, and levofloxacin.

who did not receive vancomycin prophylaxis developed CDI during alloHCT $(P<.001)$. Given the lack of cases in the intervention group, a time-to-event model could not be developed for the primary outcome. The median time to CDI diagnosis was 8 days (interquartile range, 6-12 days) in the control group. The median length of stay was similar between the 2 groups (29 vs. 28 days; $P=.22$ ). There were 3 patients -1 in the intervention group and 2 in the control group-who developed VRE bloodstream infections during their hospitalizations following alloHCT. There were no differences between the intervention and control groups in the incidences of neutropenic fever (74.4\% vs $76.4 \%$, respectively; $P=.80$ ) or bloodstream infections ( $21.1 \%$ vs $32.7 \%$, respectively; $P=.12$ ). We also examined the incidence of CDI for 90 days after the completion of oral vancomycin prophylaxis and found that $4 / 90(4.4 \%)$ of patients developed CDIs after a median of 60 days (range, 27-83 days) after vancomycin discontinuation.

\section{Graft-versus-Host Disease}

The cumulative incidence of acute, grades $2-4$ GVHD at day 180 following alloHCT was $39 \%$. In a bivariable analysis, oral vancomycin prophylaxis was not associated with a higher risk of acute, grades 2-4 GVHD (sHR 1.59; 95\% CI 0.88-2.89; $P=.12$ ). In multivariable analyses adjusting for graft sources, the relationship between oral vancomycin prophylaxis and acute, grades 2-4 GVHD remained non-significant $(P=.15)$ (Table 4). Similarly, we did not observe an association between acute, grades 3-4 (severe) GVHD by day 180 , which occurred

Table 4. Unadjusted Subhazard Ratios for Acute Graft-Versus-Host Disease (GVHD) Outcomes by Day 180 Using Competing Risks Regression With Highergrade GVHD and Death as Competing Risks

\begin{tabular}{|c|c|c|c|}
\hline & $\begin{array}{c}\text { Acute, Grades } 2-4 \text { GVHD, } \\
\text { sHR }(95 \% \mathrm{Cl})\end{array}$ & $\begin{array}{c}\text { Acute, Grade, 3-4 GVHD, } \\
\text { sHR }(95 \% \mathrm{CI})\end{array}$ & $\begin{array}{c}\text { Acute, Grades 2-4 GI GVHD, } \\
\text { sHR }(95 \% \mathrm{Cl})\end{array}$ \\
\hline Oral vancomycin & $1.59(0.88,2.89) ; P=.12$ & $0.65(0.25,1.66) ; P=.36$ & $1.95(0.93,4.07) ; P=.08$ \\
\hline Age & $0.99(0.97,1.01) ; P=.13$ & $1.00(0.96,1.05) ; P=.88$ & $0.98(0.96,1.002), P=.08$ \\
\hline Female sex & $1.14(0.68,1.93) ; P=.62$ & $0.56(0.19,1.55) ; P=.26$ & $1.39(0.76,2.57) ; P=.29$ \\
\hline Lymphoid disease & $0.67(0.34,1.30) ; P=.23$ & $0.71(0.21,2.46) ; P=.59$ & $1.09(0.54,2.19) ; P=.81$ \\
\hline Reduced intensity conditioning & $0.66(0.39,1.11) ; P=.11$ & $1.44(0.55,3.78) ; P=.46$ & $0.65(0.35,1.20) ; P=.17$ \\
\hline \multicolumn{4}{|l|}{ Donor source ${ }^{a}$} \\
\hline Unrelated & $1.48(0.77,2.86) ; P=.24$ & $0.75(0.25,2.29) ; P=.62$ & $1.27(0.59,2.77) ; P=.54$ \\
\hline Other & $1.84(0.74,4.57) ; P=.19$ & $1.81(0.47,7.01) ; P=.39$ & $2.18(0.80,5.96) ; P=.13$ \\
\hline \multicolumn{4}{|l|}{ HLA match ${ }^{b}$} \\
\hline $9 / 10$ & $1.35(0.24,7.67) ; P=.73$ & $5.89(1.04,33.4) ; P=.045$ & $2.11(0.37,12.1) ; P=.41$ \\
\hline Other & $1.53(0.72,3.27) ; P=.27$ & $2.49(0.77,8.02) ; P=.13$ & $2.03(0.89,4.61) ; P=.09$ \\
\hline \multicolumn{4}{|l|}{ HCT type ${ }^{c}$} \\
\hline Bone marrow & $0.85(0.44,1.64) ; P=.63$ & $0.78(0.22,2.81) ; P=.70$ & $0.62(0.28,1.38) ; P=.24$ \\
\hline Cord & $2.76(1.18,6.43) ; P=.02$ & $4.13(1.22,13.9) ; P=.02$ & $3.11(1.23,7.86) ; P=.02$ \\
\hline \multicolumn{4}{|l|}{ GVHD prophylaxis regimen ${ }^{d}$} \\
\hline TAC/MMF & $1.46(0.75,2.84) ; P=.27$ & $1.02(0.28,3.64) ; P=.98$ & $1.77(0.83,3.77) ; P=.14$ \\
\hline Other & $0.82(0.39,1.75) ; P=.61$ & $0.90(0.24,3.33) ; P=.87$ & $0.78(0.31,1.94) ; P=.59$ \\
\hline
\end{tabular}

Abbreviations: $\mathrm{Cl}$, confidence interval; GI, gastrointestinal; GVHD, graft-versus-host disease; HCT, hematopoietic cell transplantation; HLA, human leukocyte antigen; MMF, mycophenolate mofetil; sHR, subhazard ratio; TAC, tacrolimus.

${ }^{a}$ Reference is the related donor.

${ }^{b}$ Reference is the 10/10 HLA-match.

${ }^{\mathrm{c}}$ Reference is the peripheral blood stem cell source.

${ }^{\mathrm{d}}$ Reference is tacrolimus/methotrexate. 
in $12 \%$ of the overall cohort, and oral vancomycin prophylaxis (sHR 0.65; 95\% CI 0.25-1.66; $P=.36$ ).

As an exploratory analysis, we then examined the relationship between oral vancomycin prophylaxis and risk of any acute, grades 2-4 GVHD that involved the GI tract by day 180 post-alloHCT. The cumulative incidence of acute, grades 2-4 GI GVHD was $28 \%$. There was a trend towards a higher incidence of acute, grades 2-4 GI GVHD in the intervention group, but this association did not reach statistical significance (sHR 1.95; 95\% CI 0.93-4.07; $P=.08$ ). Similar analyses were conducted for chronic GVHD at 1 year post-alloHCT, and no associations were identified (sHR 1.70; 95\% CI 0.53-5.36; $P=.36$ ).

We repeated similar analyses for the risk of acute GVHD occurring by day 100 . The cumulative incidence of acute, grades 2-4 GVHD at day 100 was $25 \%$. Oral vancomycin prophylaxis was not associated with a risk of acute, grades $2-4 \mathrm{GVHD}$ at day 100 (sHR 1.26; 95\% CI 0.63-2.53; $P=.52$ ). We also examined the association between oral vancomycin exposure and acute, grades 3-4 GVHD. The cumulative incidence of acute, grades 3-4 GVHD at day 100 was 7.6\%. On multivariable analysis, when adjusting for graft source, oral vancomycin was associated with a reduced risk of acute, grades 3-4 GVHD at day 100 (sHR 0.27; 95\% CI 0.08-0.94; $P=.04$ ).

\section{Relapse}

Because alterations in the intestinal microbiota have been associated with mitigation of the graft-versus-tumor effect, we sought to evaluate whether oral vancomycin prophylaxis influenced a risk of disease relapse. The cumulative incidence of disease relapse was $28 \%$ at 1 year post-alloHCT. In a bivariable analysis, oral vancomycin prophylaxis was not associated with an increased risk of disease relapse (sHR 0.80; 95\% CI 0.43-1.50; $P=.50)$. In a multivariable analysis, adjusting for sex, there was no significant difference in the risk of relapse between groups.

\section{Non-relapse Mortality; Graft-versus-Host Disease-free, Relapse-free Survival; and Overall Survival}

The 1-year estimated rate of OS was 72\%. Vancomycin prophylaxis was not associated with OS (HR 0.60; 95\% CI 0.32-1.12; $P=.11$ ) or NRM (HR 0.72; 95\% CI $0.29-1.83 ; P=.49$ ). There was no significant difference in GVHD-free, relapse-free survival rates between control and intervention patients $(38.2 \%$ vs. $45.6 \%$, respectively; $P=.39$ ) at 1 year.

\section{DISCUSSION}

We demonstrated that oral vancomycin prophylaxis was highly effective in preventing CDI in alloHCT recipients, as no patient that received the intervention developed CDI, compared to a $20 \%$ CDI rate in the control group. Oral vancomycin prophylaxis was not associated with an increased risk of GVHD or disease relapse. Moreover, oral vancomycin prophylaxis did not result in an increase in VRE bloodstream infections. To the best of our knowledge, this study is the first to examine the clinical benefit of primary prophylaxis with oral vancomycin against CDI in alloHCT recipients.

Risk factors for CDI in alloHCT recipients include a prior history of CDI, myeloablative conditioning, broad-spectrum antimicrobial use, and an age greater than 60 years [15-20]. Unfortunately, the majority of CDI risk factors in the alloHCT population are not modifiable. Thus, the high incidence rate and lack of modifiable predisposing factors has created an urgent need to identify novel strategies to mitigate the risk of this infectious complication. We opted to initiate oral vancomycin prophylaxis on admission for a transplant, as the majority of CDI cases are diagnosed early in the alloHCT course. In our institution, the median time to CDI diagnosis was 8 days from admission for alloHCT, which is consistent with previous findings $[16,19]$. While we did not perform a medical records review to differentiate whether a PCR-positive test was suggestive of infection versus colonization, previous data from our institution suggest that hematologic malignancy patients with enzyme immunoassay-positive and PCR-positive tests have similar rates of signs and symptoms of infections and clinical outcomes [21].

The effectiveness of oral vancomycin for CDI prevention has been previously examined as a secondary prophylaxis approach in non-oncology settings, with encouraging findings [22-24]. However, these analyses did not include alloHCT recipients, who have a higher risk of CDI, and, thus, the results from the non-oncology setting are not generalizable. In addition, the existing literature has examined the effectiveness of oral vancomycin as a secondary prophylaxis against CDI, whereas in our study, the majority of patients were treated with oral vancomycin for primary prophylaxis. Recently, a randomized, placebo-controlled trial evaluated the role of fidaxomicin for the prevention of CDI in alloHCT recipients receiving fluoroquinolone prophylaxis and found similar prophylaxis failure rates between the 2 arms, but fewer confirmed CDI diagnoses with fidaxomicin [25]. These findings are encouraging, although a comparison of the efficacy and safety of fidaxomicin and oral vancomycin for this specific indication is not available.

We also examined whether oral vancomycin prophylaxis influenced the risk of immune-mediated processes such as GVHD and disease relapse, given the recently-described associations between a loss of intestinal microbial diversity and alloHCT clinical outcomes $[26,27]$. We did not identify an association between oral vancomycin prophylaxis and either acute GVHD at 180 days post-alloHCT or chronic GVHD at 1 year post-alloHCT. Of note, oral vancomycin was protective against severe, acute GVHD occurring by day 100, but not by day 180. The associations between oral vancomycin prophylaxis and severe, acute GVHD remain an important area for future study. In addition, we also observed a trend towards a higher incidence of acute, grades 2-4 GVHD of the GI tract in 
the intervention arm, but these data did not achieve statistical significance. The lack of association between oral vancomycin exposure and an increased risk of GVHD is noteworthy, given recent findings from several groups that have suggested that the classes of antimicrobials that are administered to alloHCT recipients may impact the risk of GVHD via effects on commensal bacteria $[28,29]$. In addition, a retrospective analysis of 857 alloHCT recipients concluded there was a higher risk of GVHD-related mortality in patients who were treated with either imipenem-cilastatin or piperacillin-tazobactam for neutropenic fever. Interestingly, the authors of this analysis did not observe a relationship between oral vancomycin exposure and GVHD-related mortality [26]. Another retrospective examination of 399 alloHCT recipients reported a higher risk of GI GVHD in patients exposed to penicillin derivatives and carbapenems [30].

The nexus between CDI, oral vancomycin, and GVHD is poorly characterized and is a very active area of research, given transplant recipients' susceptibility to intestinal dysbiosis from extensive exposure to systemic antibiotics, cytotoxic chemotherapy, and irradiation [10]. Several studies in non-oncology settings have found that oral vancomycin reduces microbial diversity via a decrease in gram-positive bacteria, as well as the gram-negative, anaerobic Bacteroides species [31-33]. However, the effects of oral vancomycin on intestinal microbial diversity in the context of an alloHCT have not been examined, and the understanding of what interconnection exists between oral vancomycin, intestinal microbiome integrity, and GVHD remains scant. Further complicating matters related to the microbiome in the context of HCT is the role of prophylactic gut decontamination, a strategy that many transplant centers continue to practice in an effort to reduce GVHD [34]. Early data suggested a reduction in GVHD with gut decontamination, but more recent evidence has shown this strategy may actually increase the risk of GVHD [35-37]. Oral vancomycin is a drug that often comprises part of the pharmacologic gut decontamination process, and how its presence affects the outcomes of gut decontamination remains unknown. Consequently, our center does not utilize gut decontamination as a GVHD reduction strategy: a recommendation consistent with consensus guidelines [38].

In our study, we did not observe an association between oral vancomycin prophylaxis and disease relapse, implying that oral vancomycin does not impair the graft-versus-tumor effect, although this was an observational study that was not powered to detect relapse differences. Nevertheless, this is an important finding given the results of a recent retrospective study that demonstrated an inverse correlation between the anaerobic, gram-positive rod Eubacterium limosum and the risk of disease relapse in alloHCT recipients [27]. The associations between intestinal microbiome integrity and the graft-versus-tumor effect remain to be determined.
There are several limitations to this analysis. AlloHCT recipients rarely receive homogeneous antibiotic therapy, which makes it challenging to robustly evaluate the effects of oral vancomycin on immune-mediated processes, such as GVHD and disease relapse, in the context of heterogeneous antimicrobial exposure. That being said, we did not identify any differences in gram-negative or gram-positive antibiotic exposure within 30 days prior to or during the time at risk after alloHCT between the 2 arms. Interestingly, more patients in the control arm received metronidazole, an active agent against CDI, prior to alloHCT. Despite a higher use of metronidazole in the control arm, the incidence of CDI was higher in this group. In addition, this was a retrospective study of sequential rather than concurrent patients. However, we could not identify any changes in our clinical practice during the time period other than the use of oral vancomycin prophylaxis for all alloHCT that may have influenced CDI rates. Also, the sample size in this study was relatively small and may not have been powered to detect differences in more rare outcomes. Moreover, the lack of difference in the length of hospitalization, incidence of GVHD, and survival outcomes between the 2 groups raises the argument of whether treating CDI early may not be worse than preventing its occurrence. Long-term follow-up in a larger number of patients will be critical to address this quandary, particularly for the latter 2 endpoints.

In summary, prophylaxis with oral vancomycin is highly effective in preventing CDI in alloHCT recipients without increasing the risk of GVHD or disease relapse. Detailed evaluation via a prospective protocol is warranted.

\section{Notes}

Acknowledgments. The authors thank Warren Bilker, $\mathrm{PhD}$, senior biostatistician in the Department of Biostatistics, Epidemiology, and Informatics at the University of Pennsylvania for his guidance with the statistical analysis portion of this study. They also thank the patients, their families, and the entire Blood and Marrow Transplant Program at the University of Pennsylvania that participated in this project.

Financial support. This work was supported by the National Institutes of Health (grant number K01-AI103028 to J. H. H.).

Potential conflicts of interest. All authors: No reported conflicts. All authors have submitted the ICMJE Form for Disclosure of Potential Conflicts of Interest. Conflicts that the editors consider relevant to the content of the manuscript have been disclosed.

\section{References}

1. Patel SS, Rybicki LA, Corrigan D, et al. Prognostic factors for mortality among day +100 survivors after allogeneic hematopoietic cell transplantation. Biol Blood Marrow Transplant 2018; 24:1029-34.

2. Schuster MG, Cleveland AA, Dubberke ER, et al. Infections in hematopoietic cell transplant recipients: results from the organ transplant infection project, a multicenter, prospective, cohort study. Open Forum Infect Dis 2017; 4:1-7.

3. Dubberke ER, Reske KA, Olsen MA, et al. Epidemiology and outcomes of Clostridium difficile infection in allogeneic hematopoietic cell and lung transplant recipients. Transpl Infect Dis 2018; 20:e12855.

4. Guddati AK, Kumar G, Ahmed S, et al. Incidence and outcomes of Clostridium difficile-associated disease in hematopoietic cell transplant recipients. Int J Hematol 2014; 99:758-65.

5. Callejas-Díaz A, Gea-Banacloche JC. Clostridium difficile: deleterious impact on hematopoietic stem cell transplantation. Curr Hematol Malig Rep 2014; 9:85-90. 
6. Alonso CD, Treadway SB, Hanna DB, et al. Epidemiology and outcomes of Clostridium difficile infections in hematopoietic stem cell transplant recipients. Clin Infect Dis 2012; 54:1053-63.

7. Trifilio SM, Pi J, Mehta J. Changing epidemiology of Clostridium difficile-associated disease during stem cell transplantation. Biol Blood Marrow Transplant 2013; 19:405-9.

8. D'Souza A, Fretham C. Current uses and outcomes of Hematopoietic Cell Transplantation (HCT): CIBMTR summary slides, 2017. Retrieved from http:// www.cibmtr.org

9. Dubberke ER, Reske KA, Srivastava A, et al. Clostridium difficile-associated disease in allogeneic hematopoietic stem-cell transplant recipients: risk associations, protective associations, and outcomes. Clin Transplant 2010; 24:192-8.

10. Shallis RM, Terry CM, Lim SH. Changes in intestinal microbiota and their effects on allogeneic stem cell transplantation. Am J Hematol 2018; 93:122-8.

11. O'Driscoll T, Crank CW. Vancomycin-resistant enterococcal infections: epidemiology, clinical manifestations, and optimal management. Infect Drug Resist 2015 8:217-30.

12. Przepiorka D, Weisdorf D, Martin P, et al. 1994 Consensus conference on acute GVHD grading. Bone Marrow Transplant 1995:825-8.

13. Filipovich AH, Weisdorf D, Pavletic S, et al. National Institutes of Health consensus development project on criteria for clinical trials in chronic graft-versushost disease: I. Diagnosis and staging working group report. Biol Blood Marrow Transplant 2005; 11:945-56.

14. Armand P, Kim HT, Logan BR, et al. Validation and refinement of the Disease Risk Index for allogeneic stem cell transplantation. Blood 2014; 123:3664-71.

15. Cannon CM, Musuuza JS, Barker AK, et al. Risk of Clostridium difficile Infection in hematology-oncology patients colonized with toxigenic C. difficile. Infect Control Hosp Epidemiol 2017; 38:718-20.

16. Aldrete SD, Kraft CS, Magee MJ, et al. Risk factors and epidemiology of Clostridium difficile infection in hematopoietic stem cell transplant recipients during the peritransplant period. Transpl Infect Dis 2017; 19:1-6.

17. Agha A, Sehgal A, Lim MJ, et al. Peri-transplant Clostridium difficile infections in patients undergoing allogeneic hematopoietic progenitor cell transplant. Am J Hematol 2016; 91:291-4.

18. Ma GK, Brensinger CM, Wu Q, Lewis JD. Increasing incidence of multiply recurrent Clostridium difficile infection in the United States: a cohort study. Ann Intern Med 2017; 167:152-8.

19. Willems L, Porcher R, Lafaurie M, et al. Clostridium difficile infection after allogeneic hematopoietic stem cell transplantation: incidence, risk factors, and outcome. Biol Blood Marrow Transplant 2012; 18:1295-301.

20. Huang AM, Marini BL, Frame D, Aronoff DM, Nagel JL. Risk factors for recurrent Clostridium difficile infection in hematopoietic stem cell transplant recipients. Transpl Infect Dis 2014; 16:744-50.

21. Ziegler M, Landsburg D, Pegues D, et al. Clinical characteristics and outcomes of hematologic malignancy patients with positive Clostridium difficile toxin immunoassay versus polymerase chain reaction test results. Infect Control Hosp Epidemiol 2018; 39:863-6.

22. Splinter LE, Kerstenetzky L, Jorgenson MR, et al. Vancomycin prophylaxis for prevention of Clostridium difficile infection recurrence in renal transplant patients. Ann Pharmacother 2018; 52:113-9.

23. Carignan A, Poulin S, Martin P, et al. Efficacy of secondary prophylaxis with vancomycin for preventing recurrent Clostridium difficile infections. Am J Gastroenterol 2016; 111:1834-40.
24. Van Hise NW, Bryant AM, Hennessey EK, Crannage AJ, Khoury JA, Manian FA. Efficacy of oral vancomycin in preventing recurrent Clostridium difficile infection in patients treated with systemic antimicrobial agents. Clin Infect Dis $\mathbf{2 0 1 6}$ 63:651-3.

25. Mullane KM, Winston DJ, Nooka A, et al. A randomized, placebo-controlled trial of fidaxomicin for prophylaxis of Clostridium difficile associated diarrhea in adults undergoing hematopoietic stem cell transplantation. Clin Infect Dis 2018 1-8. doi: 10.1093/cid/ciy484.

26. Shono Y, Docampo MD, Peled JU, et al. Increased GVHD-related mortality with broad-spectrum antibiotic use after allogeneic hematopoietic stem cell transplantation in human patients and mice. Sci Transl Med 2016; 8:1-30.

27. Peled JU, Devlin SM, Staffas A, et al. Intestinal Microbiota and Relapse After Hematopoietic-Cell Transplantation. J Clin Oncol 2017; 35:1650-9.

28. Jenq RR, Taur Y, Devlin SM, et al. Intestinal Blautia is associated with reduced death from graft-versus-host disease. Biol Blood Marrow Transplant 2015 21:1373-83.

29. Jenq RR, Ubeda C, Taur Y, et al. Regulation of intestinal inflammation by microbiota following allogeneic bone marrow transplantation. J Exp Med 2012, 209:903-11.

30. Farowski F, Bücker V, Vehreschild JJ, et al. Impact of choice, timing, sequence and combination of broad-spectrum antibiotics on the outcome of allogeneic haematopoietic stem cell transplantation. Bone Marrow Transplant 2018; 53:52-7.

31. Vrieze A, Out C, Fuentes S, et al. Impact of oral vancomycin on gut microbiota, bile acid metabolism, and insulin sensitivity. J Hepatol 2014; 60:824-31.

32. Isaac S, Scher JU, Djukovic A, et al. Short- and long-term effects of oral vancomycin on the human intestinal microbiota. J Antimicrob Chemother 2017; $72: 128-36$.

33. Lewis BB, Buffie CG, Carter RA, et al. Loss of microbiota-mediated colonization resistance to Clostridium difficile infection with oral vancomycin compared with metronidazole. J Infect Dis 2015; 212:1656-65.

34. Whangbo J, Ritz J, Bhatt A. Antibiotic-mediated modification of the intestinal microbiome in allogeneic hematopoietic stem cell transplantation. Bone Marrow Transplant 2017; 52:183-90.

35. Vossen JM, Heidt PJ, van den Berg H, Gerritsen EJ, Hermans J, Dooren LJ Prevention of infection and graft-versus-host disease by suppression of intestinal microflora in children treated with allogeneic bone marrow transplantation. Eur J Clin Microbiol Infect Dis 1990; 9:14-23.

36. Beelen DW, Haralambie E, Brandt H, et al. Evidence that sustained growth suppression of intestinal anaerobic bacteria reduces the risk of acute graft-versushost disease after sibling marrow transplantation. Blood 1992; 80:2668-76.

37. Gjærde LK, Schmidt M, Sengeløv H. Gut decontamination during allogeneic hematopoietic stem cell transplantation and the risk of acute graft-versus-host disease. Bone Marrow Transplant 2018; 53:1061-4.

38. Tomblyn M, Chiller $\mathrm{T}$, Einsele $\mathrm{H}$, et al.; Center for International Blood and Marrow Research; National Marrow Donor program; European Blood and MarrowTransplant Group; American Society of Blood and Marrow Transplantation; Canadian Blood and Marrow Transplant Group; Infectious Diseases Society of America; Society for Healthcare Epidemiology of America; Association of Medical Microbiology and Infectious Disease Canada; Centers for Disease Control and Prevention. Guidelines for preventing infectious complications among hematopoietic cell transplantation recipients: a global perspective. Biol Blood Marrow Transplant 2009; 15:1143-238. 